University of Nebraska - Lincoln

DigitalCommons@University of Nebraska - Lincoln

Sociology Department, Faculty Publications

Sociology, Department of

8-2008

Diagnostic Prevalence Rates from Early to Mid Adolescence among Indigenous Adolescents: First Results from a Longitudinal Study

\author{
Les B. Whitbeck \\ University of Nebraska-Lincoln, Iwhitbeck2@unl.edu \\ Mansoo $\mathrm{Yu}$ \\ University of Nebraska-Lincoln \\ Kurt Johnson \\ Pennsylvania State University \\ Dan R. Hoyt \\ University of Nebraska-Lincoln, dhoyt2@unl.edu \\ Melissa L. Walls \\ University of Minnesota, mlwalls@umn.edu
}

Follow this and additional works at: https://digitalcommons.unl.edu/sociologyfacpub

Part of the Sociology Commons

Whitbeck, Les B.; Yu, Mansoo; Johnson, Kurt; Hoyt, Dan R.; and Walls, Melissa L., "Diagnostic Prevalence Rates from Early to Mid Adolescence among Indigenous Adolescents: First Results from a Longitudinal Study" (2008). Sociology Department, Faculty Publications. 31.

https://digitalcommons.unl.edu/sociologyfacpub/31

This Article is brought to you for free and open access by the Sociology, Department of at DigitalCommons@University of Nebraska - Lincoln. It has been accepted for inclusion in Sociology Department, Faculty Publications by an authorized administrator of DigitalCommons@University of Nebraska - Lincoln. 
Published in Journal of the American Academy of Child and Adolescent Psychiatry 47:8 (August 2008), pp. 890-900; doi 10.1097/ CHI.0b013e3181799609 Copyright (C) 2008 by the American Academy of Child and Adolescent Psychiatry. Used by permission.

This research was funded by the National Institute of Mental Health (MH 57110) and the National Institute on Drug Abuse (DA 13580), Les B. Whitbeck, Principal Investigator. This article was the subject of an editorial by Drs. Allison Barlow and John Walkup appearing in the same August 2008 issue of JAACAP.

Accepted February 21, 2008.

\title{
Diagnostic Prevalence Rates from Early to Mid-Adolescence among Indigenous Adolescents: First Results from a Longitudinal Study
}

\author{
Les B. Whitbeck, Ph.D., ${ }^{*}$ Mansoo Yu, Ph.D., ${ }^{*}$ Kurt D. Johnson, Ph.D., ${ }^{*}$ \\ Dan R. Hoyt, Ph.D., ${ }^{*}$ and Melissa L. Walls, Ph.D.*** \\ * Department of Sociology, University of Nebraska-Lincoln \\ ** Department of Sociology, Pennsylvania State University \\ *** Department of Sociology, University of Minnesota \\ Corresponding author-L. B. Whitbeck, Department of Sociology, \\ University of Nebraska-Lincoln, 739 Oldfather Hall, Lincoln, NE 68588-0324; \\ email lwhitbeck2@unlnotes.unledu.
}

\begin{abstract}
Objective: To investigate change in prevalence rates for mental and substance abuse disorders between early and mid-adolescence among a cohort of indigenous adolescents.

Method: The data are from a lagged, sequential study of 651 indigenous adolescents from a single culture in the northern Midwest United States and Canada. At waves 1 (ages 10-12 years) and 4 (ages 13-15 years), one adult caretaker and one tribally enrolled adolescent completed a computer-assisted personal interview that included Diagnostic Interview Schedule for Children-Revised assessment for 11 diagnoses. Multivariate analyses investigate effects of wave 1 adolescent diagnosis and wave 1 biological mother diagnosis (University of Michigan Composite International Diagnostic Interview) on wave 4 diagnostic outcomes.

Results: The findings show a increase in prevalence rates for substance abuse disorders and conduct disorders between ages 10 and 12 years and 13 and 15 years among indigenous adolescents, with these disorders affecting more than one fourth of the children. The rate of lifetime conduct disorder is about twice that expected in general population studies $(23.4 \%$ versus $5 \%-$ $10 \%)$, and the rate of lifetime substance abuse disorder (27.2\%) is three times that reported in the 2004 National Survey on Drug Use and Health (9.4\%) for individuals 12 years or older. Prevalence rates for any single mental or substance use disorder (44.8 lifetime) for the 13-to 15-year-olds are similar to the lifetime prevalence rates reported in the National Comorbidity SurveyReplication (46.4\%) for individuals 18 years and older.

Conclusions: A mental health crisis exists on the indigenous reservations and reserves that participated in this study. Current service systems are overwhelmed and unable to meet the demands placed upon them.
\end{abstract}

Keywords: American Indian adolescents, prevalence of psychiatric diagnoses, mental health.

Decades of research have documented early onset of externalizing and internalizing symptoms among indigenous children. However, to date, there have been no diagnostic studies that investigate the emergence of mental and substance use disorders across time. The most cited studies of psychiatric disorders among indigenous children have been the Great Smoky Mountains Study (GSMS) ${ }^{1}$ and a study of northern Plains indigenous adolescents. ${ }^{2}$ The GSMS sample contained 323 Cherokee children ages 9, 11, and 13, years who were compared to 933 similar age European American children. The indigenous children had a slightly lower overall prevalence of psychiatric disorders than the European American children; however, although rates of substance abuse were low, as would be expected among this age group, the indigenous children were significantly more likely to meet criteria for substance abuse disorder (SUD; 1.2\%) than their European American counterparts $(0.1 \%)$. This was the only statistically signifi- 
cant difference in prevalence of psychiatric disorders between the two groups.

In the northern Plains study of 109 indigenous adolescents ages 14 to 16 years (mean 15.6 years), indigenous adolescents were more likely than European American adolescents to meet 6-month criteria for attention-deficit/hyperactivity disorder (ADHD), SUD, and conduct disorder (CD), but had similar or lower rates of major depressive episode (MDE) and anxiety disorders ${ }^{2}$ compared with adolescents in the Methodology for Epidemiology of Child and Adolescent Mental Disorders studies ${ }^{3}$ and the Oregon Adolescent Depression Project. ${ }^{4}$

In 2006 Whitbeck and colleagues ${ }^{5}$ reported prevalence rates for 11 substance use and mental disorders for a sample of 736 tribally enrolled children ages 10 to 12 years (mean 11 years). Although not strictly comparable to the GSMS, the rates for SUD, behavioral disorders, and depressive disorders were roughly twice those reported in the GSMS. Rates of ADHD were several times higher, and rates of comorbid disorders were nearly three times those reported in the GSMS. The research reported here provides prevalence data from this cohort of adolescents now ages 13 to 15 years and investigates factors associated with change in prevalence across time.

\section{Method}

\section{Procedures}

These data were collected as part of a lagged sequential study under way on four reservations in the northern Midwest and four Canadian reserves that involves yearly interviews with the adolescent and at least one primary caretaker. The data are from waves 1 (ages 10-12 years) and 4 (ages 1315 years) of the study. Wave 1 data were collected on two U.S. reservations and one Canadian reserve from February through October 2002, and from a closely related study on two U.S. reservations and three remote Canadian reserves February through October 2003. There was a similar 1-year lag between study sites for the wave 4 data collection during 2005 and 2006.

Three of the Canadian Reserves are classified as remote in that they are considerable distances from even small towns and are accessed by nonpaved roads. The reserves and reservations share a common cultural tradition and language with minor regional variations in dialects. The sample is representative of one the most populous indigenous cultures in the United States and Canada. The long-range purpose of the longitudinal study is to identify culturally specific resilience and risk factors that affect children's well being and then to use the information to guide the development of culturally based interventions.

The project was designed in partnership with the participating reservations and reserves. Before the application funding, the research team was invited to work on these reservations, and tribal resolutions were obtained. As part of our agreement to work together, the researchers promised that participating reservations would be kept confidential in published reports. On each participating reservation, an advisory board was appointed by the tribal council. The advisory boards were responsible for advising regarding difficult personnel problems, questionnaire development, and ensuring that published reports were respectful and protected the identity of the respondents and the culture. Upon advisory board consensus of the questionnaires, the study procedures and questionnaires were submitted for review by the university institutional review board for approval.

All of the interviewers on the reservations/reserves were approved by the advisory board and were either tribal members or, in a few cases (three or four of approximately 45 interviewers), nonmembers who are spouses of tribal members. Each reservation/reserve had a local indigenous full-time interviewer supervisor who coordinated visits and provided quality control, including first edits. To ensure quality of data collection, all of the interviewers underwent special training for conducting computer-assisted personal interviewing for the diagnostic measures. The training took place onsite for a period of 3 days and included practice interviews and feedback sessions regarding interview quality. Before each wave of data collection, each interviewer submitted four diagnostic interviews for feedback. By wave 4 of the study, we had a core group of highly experienced local supervisors and interviewers who were comfortable with computer-assisted personal interviewing and the diagnostic interview schedules. All of the interviewers completed annual required human subjects protection training that emphasized the importance of confidentiality and taught procedures to maintain the confidentiality of data.

Each tribe provided a list of families of enrolled children ages 10 to 12 years who lived on or near (within 50 miles) the reservation or reserve. We attempted to contact all of the families with a target child within the specified age range. Families were recruited with a personal visit by an indigenous interviewer at which time the project was explained to them. They were then presented with a traditional gift and invited to participate. If they agreed to be interviewed, then each family member received $\$ 40$ for their time when the interviews were completed. The recruitment procedure resulted in an overall response rate of $79.4 \%$. For as intrusive a design as this, we believe that the response rate was respectable. However, the most dysfunctional and substance-abusing families were likely to refuse, which may lend a conservative bias to our findings.

During the 3 years between waves 1 and 4 , the sample was reduced from 743 to 651 adolescents. The annual retention rates have been high, ranging from $93 \%$ to $100 \%$, with $88 \%$ overall retention from wave 1 to wave 4 . Of those who left the study, $6.2 \%$ of the attrition was due to refusal to participate. Other reasons for attrition included the death of the study adolescent (eight individuals), loss of contact with the family (34 families), and five unusable adolescent questionnaires. The only statistically significant difference between those who left the study and those who remained in it was that the adolescents who left the study were more likely to meet criteria for a depressive disorder at wave 1 . The multivariate analyses were based on a matched sample of biological mothers and children that further reduced the sample to 480 . 


\section{Measures}

Child diagnostic information from parents and child reports were obtained for 11 diagnoses. The substance abuse disorders (alcohol, alcohol dependence, marijuana abuse, marijuana dependence, nicotine dependence), MDE, dysthymic disorder, general anxiety disorder, oppositional defiant disorder (ODD), CD, and inattention/hyperactivity disorder modules were used from the Diagnostic Interview Schedule for Children-Revised (DISCR). Cultural sensitivity regarding any measures that would identify child maltreatment prohibited administering the posttraumatic stress disorder module. The DISC-R is a highly regarded structured interview intended for use with trained interviewers. Test-retest reliability for self-reports of children younger than 11 years varies by diagnostic category, with younger children being particularly unreliable reporters of onset and duration of symptoms. Parents tend to report more symptoms and to report them more reliably than children. Reliability of parent reports was excellent for inattention/hyperactivity and fair for overanxious disorder, ODD, and CD. ${ }^{6}$ The DISC-R has been used extensively for children 11 years and older. ${ }^{7,8}$

Reliability research on various versions of the DISC indicate that parent reports are the most reliable and that combined parent and child reports are more reliable than child reports alone. ${ }^{9,10}$ Bird and colleagues ${ }^{11}$ suggest that parents and children may each provide unique information regarding symptoms and that both sources of information are important for meaningful diagnosis. Similarly, Jenson and colleagues ${ }^{12}$ argue that although discrepant caretaker and child reports provide meaningful information in some cases (e.g., ADHD), child reports should be treated cautiously. Given the propensity of research that indicates combined reports provide the most inclusive information, we relied on combined caretaker and child reports for our multivariate analyses. If diagnostic criteria are met by either parent or child reports or if the combined reports of symptoms meet criteria, then they represent caseness in our analyses.

To reflect the considerable variation between caretaker and child reports reflected in the literature, we report caretaker, child, and combined caretaker and child prevalence rates. For the multivariate analyses, we used only the reports for matched biological mothers and their children to capture family effects. We were able to include four diagnoses for biological mothers. The number of adult diagnoses was limited by time constraints and cultural sensitivity expressed by advisory boards regarding the nature of questions for some of the diagnoses such as antisocial personality disorder and posttraumatic stress disorder. Diagnoses for the adult caretakers were from the University of Michigan Composite International Diagnostic Interview (UM CIDI). The UM-CIDI is based on DSMIII-R criteria and represents the University of Michigan revision of the CIDI used in the National Comorbidity Survey. ${ }^{13,14}$ The CIDI, ${ }^{13}$ from which the UM-CIDI is derived, is a well-established diagnostic instrument that has shown excellent interrater reliability, test-retest reliability, and validity for the diagnoses used in this study. The UM-CIDI has been used extensively with trained interviewers who are not clinicians. The version used in this study included cultural modifications similar to those in the American Indian Service Utilization, Psychiatric Epidemiology, Risk and Protective Factors Project. ${ }^{15}$

The most prevalent lifetime adult diagnosis among biological mothers was alcohol abuse (63.6\%). About $20 \%$ of the biological mothers met criteria for alcohol dependence, drug abuse, and MDE. Approximately 6\% met criteria for general- ized anxiety disorder (GAD). Diagnoses were coded " 1 " when biological mothers met diagnostic criteria and " 0 " when they did not meet criteria.

Control variables for the multivariate analyses included sex and age of child, living in a single-mother household, and living in a remote Canadian reserve. Descriptive statistics for all of the variables. in the multivariate analyses are provided in Table 1.

\section{Results}

The prevalence of meeting criteria for a single lifetime disorder increased from $25.6 \%$ (combined caretaker and child report) at wave 1 when the children were ages 10 to 12 years to $44.8 \%$ at wave 4 when the children had reached 12 to 15 years (Table 2). The prevalence of meeting criteria for any single 12-month disorder increased from $23.4 \%$ at wave 1 to $41.2 \%$ at wave 4 . Rates of lifetime comorbidity among the 13 disorders increased from $9.2 \%$ to $26.6 \%$.

\section{Substance Use Disorders}

The prevalence of SUDs rose dramatically between early and mid-adolescence. At wave 1 , the prevalence of any lifetime SUD was 3.2\%. This increased more than eight times to $27.2 \%$ by wave 4 . The largest increases were for lifetime alcohol abuse (1.1\%-13.8\%) and marijuana dependence $(1.4 \%-12.4 \%)$. Nicotine dependence increased from $1.7 \%$ to $9.5 \%$ and alcohol dependence rose from $0.5 \%$ to $9.5 \%$. Substance abuse and dependence other than alcohol, marijuana, and nicotine remained low at mid-adolescence, at about $1 \%$.

\section{Internalizing Disorders}

Twelve-month MDEs more than doubled between waves 1 and 4 , from $3.2 \%$ to $7.8 \%$. The prevalence of dysthymic disorder was negligible $(<1 \%)$. Rates for GAD increased slightly from early to mid-adolescence, from $3.4 \%$ to $4.6 \%$.

Table 1. Descriptive Information for the Multivariate Analysis at Wave 1: Wave 4 Matched Biological Mothers and Adolescents $(N=480)$

\begin{tabular}{lc}
\hline & Mean (SD) or \% \\
\hline Adolescent demographics & 54.3 \\
Female & $14.3(0.9)$ \\
Age & 8.9 \\
Remote & 30.4 \\
Single mother & \\
Mother's psychiatric disorders & 69.9 \\
Any diagnosis (lifetime) & 37.8 \\
Any more than two diagnoses (lifetime) & \\
\hline
\end{tabular}

${ }^{a}$ Canadian First Nation reserves.

${ }^{b}$ For more details regarding parent/caretaker psychiatric diagnoses, see Whitbeck et al. ${ }^{17}$ 
Table 2. Prevalence of DSM-IV Disorders

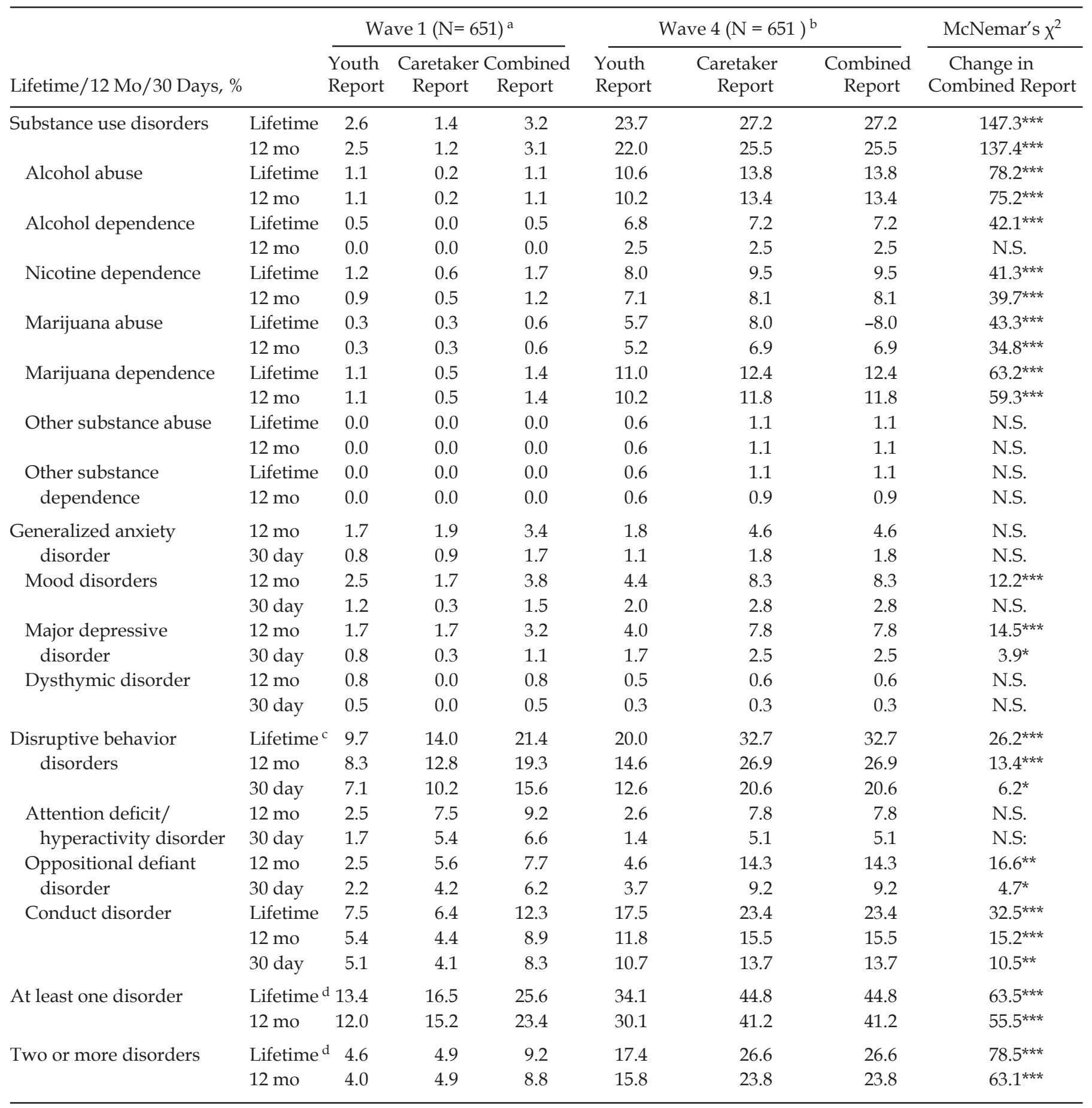

Note: NS = not significant.

a Age mean $(\mathrm{SD})=11.1(0.8)$.

${ }^{\mathrm{b}}$ Age mean $(\mathrm{SD})=14.3(0.9)$.

${ }^{\mathrm{c}}$ Lifetime (conduct disorder) + 12-month (attention deficit/hyperactivity disorder and oppositional defiant disorder).

${ }^{\mathrm{d}}$ Lifetime (all alcohol/substance use disorders and conduct disorder) + 12-month (generalized anxiety disorder, mood disorders, attention deficit/ hyperactivity disorder, and oppositional defiant disorder).

${ }^{*} p<.05 ;{ }^{* *} p<.01 ;{ }^{* * *} p<.001$. 


\section{Externalizing Disorders}

Among the externalizing disorders, the number of adolescents who met 12-month criteria for ADHD decreased from $9.2 \%$ at wave 1 to $7.8 \%$ at wave 4 . The rates of 12 -month ODD essentially doubled from $7.7 \%$ to $14.3 \%$. Twelve-month CD also nearly doubled from $8.9 \%$ to $15.5 \%$. The number of adolescents who met lifetime criteria for CD increased from $12.3 \%$ to $23.4 \%$.

\section{Caretakers versus Adolescent Reports}

By wave 4, the adolescents were more likely than caretakers to report externalizing symptoms such as substance abuse and dependence, ADHD, and CD. Caretakers and adolescents were about equally likely to report depressive disorders; however, caretakers were more likely than adolescents to report anxiety symptoms, ADHD, and ODD. These differences between adult and child reporters are congruent with those found in general populations studies. ${ }^{6}$ Caretaker observers may be more aware of ADHD, ODD, and anxiety behaviors than adolescents and hence are more reliable reporters. ${ }^{6,11,12}$ Conversely, some SUD and CD behaviors may be largely hidden from caretakers.

\section{Impairment}

Each DISC-R disorder has six impairment criteria that are rated on a 3-point scale: "hardly ever," "some of the time" (intermediate), and "a lot of the time" (severe). Based on the DISC-R algorithms of impairment, we computed three levels of impairment rates based on combined caretaker-adolescent reports for wave 4, 12-month prevalence rates for all of the diagnoses: (1) at least one intermediate impairment, (2) two or more intermediate impairments, and (3) at least one severe impairment (Table 3). Of the $25.5 \%$ of adolescents who met criteria for an alcohol/substance use disorder, $18.2 \%$ reported at least one intermediate impairment, $12.8 \%$ two intermediate impairments, and $12.4 \%$ at least one severe impairment. Nearly all of the adolescents who met criteria for 12month MDE $(7.8 \%)$ reported at least one intermediate impairment $(7.5 \%)$, and $5.5 \%$ reported at least one severe impairment. Similarly, the majority of those who met criteria for 12-month CD $(15.5 \%)$ reported at least one intermediate impairment (12.3\%), and $6.1 \%$ reported at least one severe impairment. For all of the disruptive disorders, the level 1 impairment rates $(24 \%)$ were similar to the 12 -month prevalence rates $(26.9 \%)$.

\section{Multivariate Analyses}

We did three separate logistic regression analyses to investigate correlates of wave 4 SUDs, internalizing disorders, and externalizing disorders. To ensure that we would be accounting for familial effects, the sample for the multivariate analysis included only

Table 3. Rates of Impairment at Wave $4(N=651)$ : Combined Youth and Adult Report ${ }^{a}$

\begin{tabular}{|c|c|c|c|}
\hline \multirow[b]{2}{*}{$12 \mathrm{Mo}, \%$} & \multicolumn{3}{|c|}{ Impairment Level } \\
\hline & $\begin{array}{l}\text { Least } \\
\text { rmediate } \\
\text { Rating }\end{array}$ & $\begin{array}{c}\text { At Least } \\
2 \text { Intermediate } \\
\text { Ratings }\end{array}$ & $\begin{array}{c}\text { At Least } \\
1 \text { Severe } \\
\text { Rating }\end{array}$ \\
\hline $\begin{array}{l}\text { Substance use } \\
\text { disorders }\end{array}$ & 18.2 & 12.8 & 12.4 \\
\hline Alcohol abuse & 10.4 & 8.4 & 7.5 \\
\hline Alcohol dependence & 1.5 & 0.9 & 0.8 \\
\hline $\begin{array}{l}\text { Nicotine } \\
\text { dependence }\end{array}$ & 4.3 & 1.1 & 2.0 \\
\hline Marijuana abuse & 4.4 & 2.2 & 2.8 \\
\hline $\begin{array}{l}\text { Marijuana } \\
\text { dependence }\end{array}$ & 7.5 & 4.4 & 4.3 \\
\hline $\begin{array}{l}\text { Other substance } \\
\text { abuse }\end{array}$ & 0.3 & 0.3 & 0.0 \\
\hline $\begin{array}{l}\text { Other substance } \\
\text { dependence }\end{array}$ & 0.8 & 0.6 & 0.6 \\
\hline $\begin{array}{l}\text { Generalized anxiety } \\
\text { disorder }\end{array}$ & 4.4 & 4.3 & 2.8 \\
\hline Mood disorders & 7.7 & 7.1 & 5.7 \\
\hline $\begin{array}{l}\text { Major depressive } \\
\text { disorder }\end{array}$ & 7.5 & 6.9 & 5.5 \\
\hline Dysthymic disorder & 0.3 & 0.3 & 0.3 \\
\hline $\begin{array}{l}\text { Disruptive behavior } \\
\text { disorders }\end{array}$ & 24.0 & 18.9 & 14.0 \\
\hline $\begin{array}{l}\text { Attention } \\
\text { deficit/hyperactivity } \\
\text { disorder }\end{array}$ & $\mathrm{y}^{7.7}$ & 7.2 & 5.4 \\
\hline $\begin{array}{l}\text { Oppositional } \\
\text { defiant disorder }\end{array}$ & 13.7 & 12.9 & 8.3 \\
\hline Conduct disorder & 12.3 & 5.2 & 6.1 \\
\hline
\end{tabular}

Note: Intermediate rating indicates endorsing "some of the time" in response to one of the six criteria. "Severe" rating indicates endorsing "a lot of the time" in response to one of the six criteria.

a Six impairment criteria:

1)Annoyed or upset you in the last year.

2) Kept you from doing things or going places with your family.

3) Kept you from doing things or going places with other children/youths.

4) Made problems with school work or work.

5) Caused your teachers or boss to be annoyed or upset with you.

6) Made you feel bad or upset when the problems were worst. 
matched children and biological mothers $(n=480)$. The models included demographic control variables (sex, age, remote location, and single-mother household), biological mother meeting lifetime criteria for SUD or an internalizing disorder (MDE, dysthymic disorder, or GAD) at wave 1, and combined caretaker and child reports of child diagnoses at wave 1 (Table 1). Multiplicative interactions with sex of adolescent and maternal lifetime diagnosis were checked with each independent variable.

\section{SUDs}

Age was strongly associated with meeting 12month criteria for SUD at wave 4 (Table 4). For each 1 -year increase in age, the likelihood of meeting 12month criteria for SUD rose 1.72 times. If the adolescent's biological mother met lifetime criteria for an internalizing disorder, then the likelihood that the adolescent would meet lifetime criteria for an SUD almost doubled (OR 1.93). SUD persisted over time. If the adolescent met criteria for SUD at wave 1, then he or she was 8.65 times more likely to meet 12-month SUD criteria at wave 4 than adolescents who did not meet criteria for SUD at wave 1 . There were no statistically significant multiplicative interactions with sex or maternal lifetime diagnosis for any of the variables in the model.

\section{Internalizing Disorders}

At wave 4, adolescent girls were twice (OR 2.01) as likely to meet 12-month criteria for one of the internalizing disorders (GAD, dysthymic disorder, MDE) than were boys. Having a biological mother who met wave 1 criteria for a SUD increased the odds of the adolescent meeting 12-month criteria for an internalizing disorder 2.5 times. Adolescents who met criteria for an internalizing disorder at wave 1 were greater than four times (OR 4.28) more likely to meet 12month criteria for an internalizing disorder at wave 4 than adolescents who did not meet criteria for an internalizing disorder at wave 4 . There were no statistically significant multiplicative interactions with sex or maternal lifetime diagnosis for any of the variables in the model.

\section{Externalizing Disorders}

As noted in Table 2, nearly one third of the adolescents $(32.7 \%)$ met lifetime criteria and more than one fourth $(26.9 \%)$ met 12 -month criteria for an externalizing disorder at wave 4 of the study. None of the control variables was associated with meeting 12-month criteria for an externalizing disorder (ADHD, ODD, CD) at wave 4. However, the biological mother's SUD and internalizing disorders were significantly associated with wave 4 externalizing disorders. Maternal SUD nearly doubled (OR 1.96) the likelihood that the adolescent would meet 12-month criteria for an externalizing disorder and maternal internalizing disorders increased the likelihood 1.72 times. Because the interaction between the two variables was nonsignificant and they therefore have independent effects, we can multiply the odds ratios to indicate their cumulative influence. That is, if a biological mother met criteria for both SUD and an internalizing disorder the odds of her child meeting the criteria for an externalizing disorder would be 1.96 multiplied by 1.72 or 3.37 times. If the adolescent met 12-month criteria for internalizing disorder at wave 1 , he or she was nearly 2.5 times more likely to meet 12-month criteria for an externalizing disorder at wave 4. Meeting lifetime criteria for an externalizing disorder at wave 1 doubled (OR 2.03) the odds of meeting 12-month criteria at wave 4 . There were no statistically significant multiplicative interactions with sex or maternal lifetime diagnosis for any of the variables in the model.

\section{Discussion}

Although we have no indigenous comparison groups in this age range (13-15 years), we can compare the adolescents to current prevalence rates in the general population. In the National Comorbidity Survey Replication, the lifetime prevalence for psychiatric disorder (ages 18 and older) was $46.4 \%$ for one disorder and $27.7 \%$ for two or more disorders. ${ }^{16}$

The American Indian Service Utilization, Psychiatric Epidemiology, Risk and Protective Factors Project reported a lifetime prevalence rate of one disorder of $41.9 \%$ in a southwestern culture and $44.5 \%$ in a northern Plains culture among indigenous adult samples ages 15 to 54 years. ${ }^{15}$ Among the caretaker adults in wave 1 of this study, we found a prevalence rate of $43 \%$ for one lifetime disorder and $31.6 \%$ met criteria for two or more of five disorders (alcohol abuse, alcohol dependence, drug abuse, MDE, GAD). Our findings for the 13- to 15-year-olds are similar (44.8\% lifetime; $26.6 \% 12$ months) to the adult prevalence rates reported in all of these adult samples. ${ }^{17}$ 


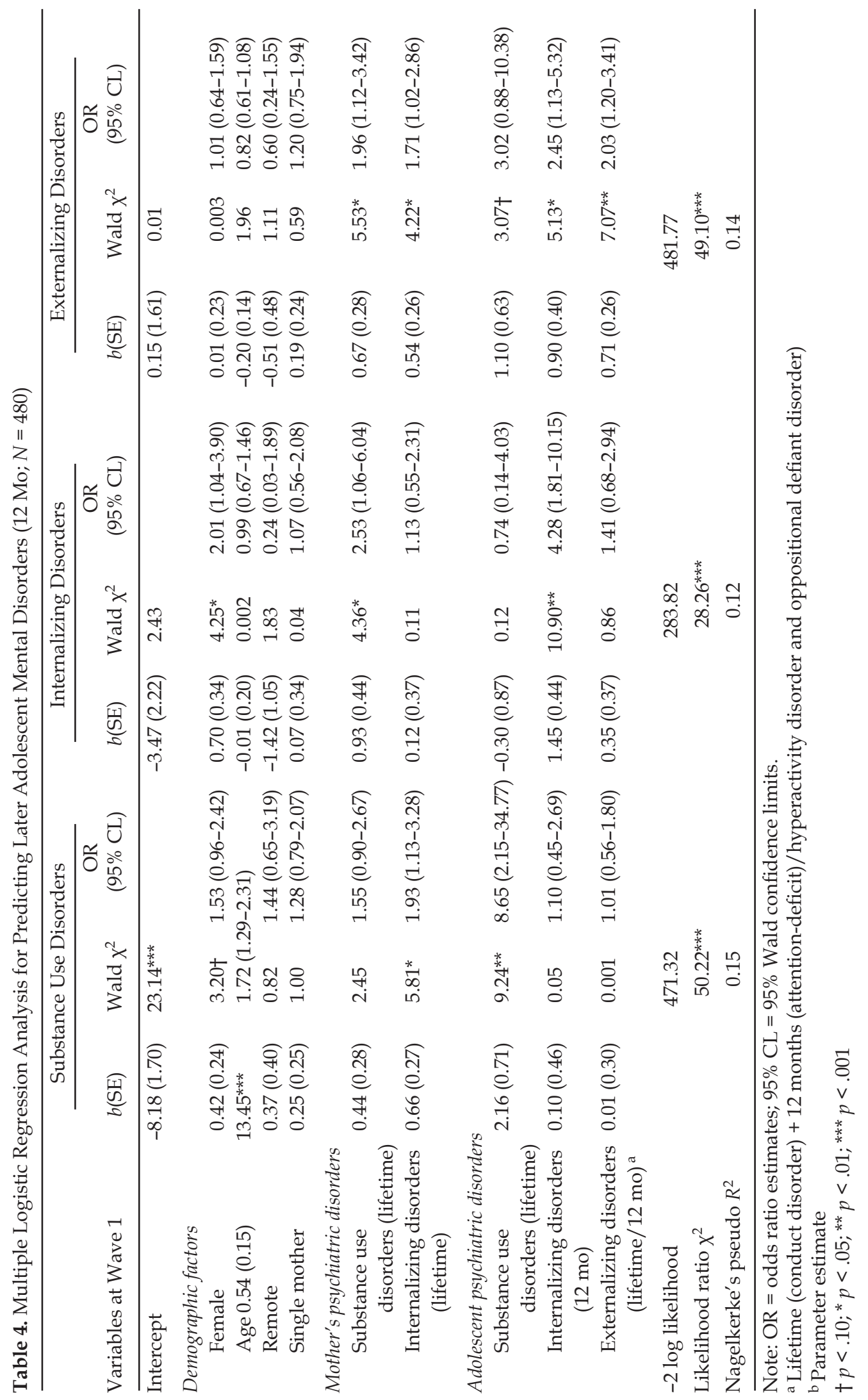


The high rates of psychiatric disorders are reflected in atypically high rates of SUD and externalizing disorders among the wave 4 adolescents. Estimates of CD for adolescents in Western industrialized countries ages 8 to 16 years are between $5 \%$ and $10 \%{ }^{18,19}$ The rates that we are reporting for lifetime CD (23.4\%) are more than twice the highest rates expected in the general population. Similarly, SUD rates among adolescents are higher than those found in general population surveys. For example, the 2004 National Survey on Drug Use and Health estimated that $9.4 \%$ of the population who were 12 years or older met the criteria for substance dependence or abuse in the past year. ${ }^{20}$ The 12 -month SUD rate $(25.5 \%)$ reported in this study is nearly three times that of the National Survey on Drug Use and Health. Rates of lifetime alcohol abuse among adolescents range from $0.4 \%$ in the Great Smoky Mountains Study ${ }^{1}$ to $9.6 \%$ in the National Comorbidity Study. ${ }^{21}$ The lifetime rate for this study of 13to 15 -year-olds is $13.8 \%$. General population rates of lifetime alcohol dependence range from $0.6 \% 1$ to $4.3 \%$ in the Oregon Adolescent Depression Project. ${ }^{4}$ The lifetime prevalence of alcohol dependence in the present study is $7.2 \%$. The lifetime prevalence of drug abuse or dependence in general population studies ranges from $3.3 \%$ in 15 -year-olds to $9.8 \%$ in 17- to 19-year- olds. ${ }^{22-24}$ The lifetime marijuana dependence rate for this cohort of adolescents is $12.4 \%$; however, rates of abuse and dependence of drugs other than marijuana are low.

General population studies of depression among children and adolescents younger than 18 years yield prevalence rates ranging between $1.6 \%$ and $8.9 \% .{ }^{25}$ The rates of 12-month MDE (7.2\%) for the wave 4 adolescents were well within that range. Prevalence rates from population studies for childhood GAD range from $0.6 \%$ to $4.2 \%$ with a median of $2 \% .{ }^{26}$ The 12 -month rates among this cohort of adolescents $(4.6 \%)$ fall at the high end of the reported range.

Impairment rates indicate at least moderate impairment for the majority of adolescents who met 12-month diagnostic criteria. Severe impairment decreased to about one half of those who met 12-month criteria. These impairment estimates suggest that the prevalence rates represent serious deficits in psychosocial functioning at a critical stage in adolescents' development.

According to Kessler and colleagues, ${ }^{16}$ estimates of age at onset vary widely by type of disorder. For example, they estimate the median age at onset for anxiety disorders and impulse-control disorders at 11 years, SUDs at 20 years, and mood disorders at 30 years. Of particular interest here, "the median age of onset was earlier for each impulse-control disorder (7-15 years) than for any substance (age 19-23 years) or mood (age 25-32 years) disorder" (p. 595). In contrast to the National Comorbidity Survey Replication estimates, the 13- to 15-year-old adolescents in our sample are manifesting about the same lifetime rates of externalizing disorders and SUDs. They are within the age range set by Kessler and colleagues for the onset of externalizing disorders but 4 to 8 years earlier for onset of SUDs. They are also well below the estimates of Kessler et al. for median age at onset for MDE.

The prevalence rates suggest two critically important trends among indigenous children in mid-adolescence. First, the high percentage of SUD and CD children portend difficult late adolescent and early adult adjustment for about one fourth of the young people. This is complicated by the fact that these are likely to be dual-diagnosis children. Comorbidity for lifetime CD with any lifetime SUD (combined caretaker and child reports) was $62 \%$. These are the adolescents who are likely to perform poorly in school, who are at risk for leaving school early, and who are likely to engage in risky sexual behaviors. They are also likely to be involved in the criminal justice system and at risk for intentional and unintentional injuries. The reservations/reserves in the study are small, economically disadvantaged, and lack sufficient health, education, and social services resources to cope with such large numbers of severely troubled adolescents.

Second, those who met criteria for SUD and MDE were significantly "off time" for age at onset. The early onset of SUDs particularly may augur later-life substance abuse problems and perhaps the transition in later adolescence and early adulthood to harder drugs or polydrug use. ${ }^{27}$ Early-onset MDE tends to recur later during adolescence and in adulthood. ${ }^{28}$ Indeed, Kovacs found that 5 years after the first depressive episode, $72 \%$ had a recurrence. ${ }^{29}$ Numerous studies have shown a relation between childhood onset of depression and adult depressive episodes. ${ }^{30-32}$ We are already noticing the effects of early disorders on later disorders in the multivariate analyses with odds ratios for preexisting disorders ranging from 2.03 to 8.65 . 
Moreover, the multivariate analyses suggest intergenerational continuity of mental and substance use disorders. Having a biological mother who met lifetime criteria for SUD or an internalizing disorder about doubles the odds of her child meeting criteria for a psychiatric disorder. If the mother was comorbid for SUD and an internalizing disorder at wave 1, then the odds that her child met criteria for an externalizing disorder at wave 4 increased to more than three times. Families in which the mother has a history of psychiatric disorder will likely to be those that will have the most difficulty coping with a child with a psychiatric disorder. The effects of social location are cumulative. Reservations lack resources to respond the high numbers of emotional and behavioral problems among the adolescents, and the families in which they reside may well be those that are the least able to cope without support.

\section{Cultural Context of Development}

Growing up on a reservation/reserve represents a unique developmental context for these adolescents historically and socially. For indigenous nations lucky enough not to be completely removed from their home territory, reservations/reserves represent the remnant "homeland." However, this "homeland" often occupies the least productive, least desirable area of what was once their vast territory. Contemporary reservations are often economically disadvantaged, rural, and socially isolated from surrounding communities. As a social context, reservations/reserves are at once a symbol of what was and the representation of what has occurred. Simply living on reservations/reserves can be a reminder of ethnic cleansing, broken promises, continual encroachment on tribal lands, and continuing pressures of assimilation. At the same time, reservations/reserves may be a refuge from discrimination and the land a symbol of the living culture.

The adolescents in this study were subject to all of the known risk factors associated with economic disadvantage. However, there are also culturally unique protective factors such as traditional spirituality, traditional extended family and community support, and community elders available to them that are often overlooked in majority research. This is a unique cultural context that is not well understood by majority researchers and practitioners. More work needs to be done to identify and understand the interplay between majority and culturally specific risk and protective factors. We urgently need this work to guide therapeutic and prevention interventions.
The major limitation of this research is the sensitivity of the DISC-R and UM-CIDI measures for assessing American Indian and Canadian First Nations people. For example, definitions of depression may vary across indigenous cultures, ${ }^{33}$ and drinking patterns among indigenous adults may include sporadic binge drinking at irregular intervals that may not be represented adequately by diagnostic criteria. ${ }^{34}$ However, this study uses the same measures as recent population studies of American Indian people to provide the best estimates possible. ${ }^{15}$

A related measurement issue is that cultural variations in the expression of some symptoms, particularly internalizing symptoms, may be poorly represented in diagnostic interview schedules. ${ }^{33,34}$ Externalizing symptoms, however, are more overt and easier to, identify. This may lead to underestimating the prevalence of internalizing disorders in comparison to externalizing disorders.

Although we interviewed a broad range of American Indian and Canadian First Nations adults and children on multiple reservations and Canadian reserves that are dispersed geographically across two Midwestern states and one Canadian province, these results pertain to a single culture and capture variations within this culture. We believe that the findings represent the culture well, but they cannot be generalized to other indigenous cultures. However, if we are to obtain sound psychiatric epidemiological information pertaining to indigenous people, then it will be necessary to proceed nation by nation with comparable measures. ${ }^{35}$

A final concern is that this report is limited to 11 diagnoses for children and only four diagnoses for the biological mothers. This limitation was dictated by time, subject burden, and sensitivity of the nations to certain diagnostic questions (e.g., antisocial personality disorders, posttraumatic stress disorder, psychoses).

This research has important clinical and policy implications for indigenous communities. It is the only diagnostic longitudinal study of which we are aware that addresses change in prevalence rates from early to mid-adolescence among indigenous youths. The findings show a dramatic increase in prevalence rates for SUD and CD between ages 10 and 12 years and 13 and 15 years among indigenous adolescents, with these disorders affecting more than one fourth of the children. We have long known that indigenous adolescents begin substance use earlier than their counterparts from other ethnic groups. ${ }^{36}$ This research charts the trajectory of those most at risk. 
These results call attention to the critical need for mental health services on indigenous reservations and reserves. These numbers would overwhelm any pediatric health system, but these communities are among the least prepared in the nation to respond effectively. Indeed, these findings are one more blow to communities that are struggling to overcome the psychological effects of 300 years of systematic ethnic cleansing. Empirical evidence is beginning to emerge linking historical losses to mental health and substance use symptoms of indigenous people. ${ }^{37}$ Such high rates of mental and substance abuse disorders are indicative of this historical legacy.

Few services exist to address these mental health disparities, distances are great, there is distrust about confidentiality, and there are few well-trained mental health workers on the reservations and reserves and almost none of these are indigenous. As we have reported elsewhere, parents do not have a high regard for existing reservation/reserve mental health and SUD services and prefer traditional helpers to those within the formal health care system. As mental health systems on the reservations/reserves evolve, it will be important to include traditional healers and mental health workers who are knowledgeable and sensitive to traditional cultures. ${ }^{38}$

The findings also indicate the need for parent and teacher training in structuring and providing limits for children and adolescents manifesting early disruptive behaviors. The prevalence of $\mathrm{CD}$ at wave 1 of the study was just a little higher than that found in general population studies, but doubled in the following 3 years. It may be that we have a window of opportunity during early adolescence to prevent the rapid increase in serious behavior problems through family and school interventions. We must work to understand the specific mechanisms that contribute to this upsurge in disruptive behaviors. The odds ratios in multivariate analyses for wave 1 SUD and CD on wave 4 SUD and CD attest to the importance of identifying and treating those with early-onset problem behaviors. The strong effects of maternal psychopathology on child outcomes also denote the risks of intergenerational effects and the need for effective family interventions.

Such high rates of mental and substance use disorders at these early ages should alarm state and national mental health policymakers. Tribal members see the consequences daily in early drug use, violence, suicides, and unintentional injuries among their young people. These findings attest to their sense of urgency for the health and safety of the next generation and the need for immediate policy changes to address the mental health disparities associated with rural reservation life.

\section{Disclosure}

The authors report no conflicts of interest.

\section{References}

1. Costello J, Farmer E, Angold A, et al. Psychiatric disorders among American Indian and white youth in Appalachia: the Great Smoky Mountains Study. Am J Public Health. 1997;87:827-832.

2. Beals J, Piasecki J, Nelson S, et al. Psychiatric disorder among American Indian adolescents: prevalence in Northern Plains youth. J Am Acad Child Adolesc. Psychiatry. 1997;36:1252-1259.

3. Shaffer D, Fisher P, Dulcan M, et al. The NIMH Diagnostic Interview Schedule for Children (DISC-2): description, acceptability, prevalences and performance in the MECA study. J Am Acad Child Adolesc. Psychiatry. 1996;35:865-877.

4. Lewinshon PM, Hops H, Roberts SE, Seeley JR, Andrews JA. Adolescent psychopathology: I. Prevalence and incidence of depression and other DSM-III disorders in high school students. J Abnorm Psychol. 1993; 102:122-144.

5. Whitbeck L, Johnson K, Hoyt D, Walls M. Prevalence and comorbidity of mental disorders among American Indian children in the northern Midwest. J Adolesc Health. 2006;39:427-434.

6. Schwab-Stone M, Fallon T, Briggs M, et al. Reliability of diagnostic reporting for children aged 6-11 years: a test-retest study of the Diagnostic Interview Schedule for Children-Revised. Am J Psychiatry. 1994;151:1048-1054.

7. Shaffer D, Schwab-Stone M, Fisher P, et al. The Diagnostic Interview for Children-Revised Version (D(SC-R): I. Preparation, field testing, interrater reliability and acceptability. J Am Acad Child Adolesc. Psychiatry. 1993;32:643-650.

8. Shaffer D, Schwab-Stone M, Fisher P, et al. A Revised Version of the Diagnostic Interview Schedule for Children (DISCR): Results of a Field Trial and Proposals for a New Instrument (DISC-2). Rockville, MD: Epidemiology and Psychopathology Research Branch, Division of Clinical Research, National Institute on Mental Health; 1988.

9. Schwab-Stone M, Shaffer D, Dulcan M, et al. Criterion validity of the NIMH Diagnostic Interview Schedule for Children version 2.3 (DISC-2.3). J Am Acad Child Adolesc. Psychiatry. 1996:35:878-888.

10. Shaffer D, Fisher P, Dulcan M, et al. The NIMH Diagnostic Interview Schedule for Children (DISC-2): description, acceptability, prevalences and performance in the MECA study. J Am Acad Child Adolesc. Psychiatry. 1996;35:865-877.

11. Bird H, Gould M, Staghezza B. Aggregating data from multiple informants in child psychiatry epidemiological research. J Am Acad Child Adolesc. Psychiatry. 1992;31:78-85.

12. Jensen P, Rubio-Stipec M, Canino G, et al. Parent and child contributions to diagnosis of mental disorder: are both informants always necessary? J Am Acad Child Adolesc. Psychiatry. 1999;38:1569-1579. 
13. Kessler R. The National Comorbidity Survey of the United States. Int Rev Psychiatry. 1994;6:365-376.

14. Kessler R. Building on the ECA: the National Comorbidity Survey and the children's ECA. Int J Methods Psychiatr Res. 1994;4:81-94

15. Beals J, Manson S, Whitesell N, et al. Prevalence of DSMIV disorders and attendant help-seeking in 2 American Indian reservation populations. Arch Gen Psychiatry. 2005;62:99-108.

16. Kessler R, Bergland P, Demler O, et al. Lifetime prevalence and age of onset distributions of DSM-IV disorders in the National Comorbidity Survey replication. Arch Gen Psychiatry. 2005;62:593-602.

17. Whitbeck L, Hoyt D, Johnson K, Chen X. Mental disorders among parents/caretakers of American Indian early adolescents in the northern Midwest. Soc Psychiatry Psychiatr Epidemiol. 2006;41:632-640.

18. Angold A, Costello EJ. The epidemiology of disorders of conduct: nosological issues and comorbidity. In: Hill J, Maugham B, eds. Conduct Disorders in Childhood and Adolescence. Cambridge, UK: Cambridge University Press; 2001:126-168.

19. America Psychiatric Association. Diagnostic and Statistical Manual of Mental Disorders, 4th edition, text revision (DSMIV-TR). Washington, DC: American Psychiatric Association; 2000.

20. Substance Abuse and Mental Health Services Administration. Results from the 2004 National Survey on Drug Use and Health: National Findings (NSDUH Series H-28, DHHS Publication No. SMA 054062). Rockville, MD: Office of Applied Studies; 2005.

21. Kessler RC, McGonagle KA, Zhao S, et al. Lifetime and 12month prevalence of DSM-III-R psychiatric disorders in the United States: Results from the National Comorbidity Study. Arch Gen Psychiatry. 1994;51:8-19.

22. Kashini JH, Beck NC, Heoper EW, et al. Psychiatric disorders in a community sample of adolescents. Am J Psychiatry. 1987; 144:584-589.

23. Reinherz HZ, Giaconia RM, Lefkowitz ES, Pakiz B, Frost AK. Prevalence of psychiatric disorders in a community population of older adolescents. J Am Acad Child Adolesc. Psychiatry. 1993;32:367-377.

24. American Academy of Child and Adolescent Psychiatry. Practice parameter for the assessment and treatment of children and adolescents with substance abuse disorders. 2005. http://www.aacap.org/galleries/PracticeParameterslsubstanceUseDisorder.pdf Accessed February 22, 2008.

25. Angold A, Costello EJ. The epidemiology of depression in children and adolescents. In: Goodyer IM, ed. The Depressed Child and Adolescent. 2nd ed. Cambridge, UK: Cambridge University Press; 2001:143-178.
26. Verhurst FC. Community and epidemiological aspects of anxiety disorders in children. In: Silverman WK, Treffers PD, eds. Anxiety Disorders in Children and Adolescents. Cambridge, UK: Cambridge University Press; 2001:273-292.

27. Kandel DB. Stages and Pathways of Drug Involvement. Cambridge, UK:, Cambridge University Press; 2002.

28. Harrington R, Dubicka B. Natural history of mood disorders in children and adolescents. In: Goodyer IM, ed. The Depressed Child and Adolescent. 2nd ed. Cambridge, UK: Cambridge University Press; 2001:353-381.

29. Kovacs M, Akiskal HS, Gatsonis C, et al. Childhood onset dysthymic disorder. Clinical features and prospective naturalistic outcome. Arch Gen Psychiatry. 1994;51:365-374.

30. Harrington RC, Fudge H, Rutter M, et al. Adult outcomes of childhood. and adolescent depression: 1. Psychiatric status. Arch Gen Psychiatry. 1990;47:465-473.

31. Lewinsohn PM, Rhode P, Klein DN, et al. Natural course of adolescent major depressive disorder: 1. Continuity into young adulthood. J Am Acad Child Adolesc. Psychiatry. 1999;38:56-63.

32. Ran U, Ryan ND, Birmaher B, et al. Unipolar depression in adolescence:, clinical outcome in adulthood. J Am Acad Child Adolesc. Psychiatry. 1995; 34:566-578.

33. Manson S. Culture and major depression: current changes in the diagnosis of mood disorders. Cultural Psychiatry. 1995;18:487-501.

34. Manson S, Shore J, Boom J. The depressive experience in American Indian communities: a challenge for psychiatric theory and diagnoses. In: Kleinman A, Good B, eds. Culture and Depression: Studies in the Anthropology and CrossCultural Psychiatry of Affect and Disorder. Berkeley: University of California Press; 1985:331-368.

35. Beals J, Manson S, Mitchell C, et al. Cultural specificity and comparison in psychiatric epidemiology: walking the tightrope in American Indian Research. Cult Med Psychiatry. 2003;27:259-289.

36. May P. Overview of alcohol abuse epidemiology for American Indian populations. In: Sandefur G, Rindfuss R, Cohen B, eds. Changing Numbers, Changing Needs: American Indian Demography and Public Health. Washington, D6 National Academy Press; 1996:235-261.

37. Whitbeck L, Chen X, Hoyt D, Adams G. Discrimination, historical loss and enculturation: culturally specific risk and resiliency factors for alcohol abuse among American Indians. J Alcohol Stud. 2004;65:409-418.

38. Walls M, Johnson K, Whitbeck L, Hoyt D. Mental health and substance abuse services preferences among American Indian people of the northern Midwest. Community Mental Health J. 2006;42:521-535. 